\title{
Traversing the Spectrum of Non-Langerhans Cell Histiocytosis: A Case of Rosai-Dorfman Disease with Features of Necrobiotic Xanthogranuloma
}

\author{
Blythe N. Ke, MD, MBA, ${ }^{1}$ Erika Belinda T. Chen, MD, ${ }^{2}$ Kevin Jer V. David, MD, ${ }^{3}$ Sarah Faye V. Obbus, MD, ${ }^{1}$ \\ Val Constantine S. Cua, MD, ${ }^{1}$ Ma. Lorna F. Frez, MD ${ }^{1}$ and Eileen A. Cubillan, MD ${ }^{1}$ \\ ${ }^{1}$ Department of Dermatology, Philippine General Hospital, University of the Philippines Manila \\ ${ }^{2}$ Division of Hematology, Department of Medicine, Philippine General Hospital, University of the Philippines Manila \\ ${ }^{3}$ Department of Otorhinolaryngology, Philippine General Hospital, University of the Philippines Manila
}

\begin{abstract}
Introduction. Non-Langerhans cell histiocytoses (non-LCH) are a group of rare diseases with varied clinical manifestations and overlapping features seen among the subtypes. Here, we present a case of Rosai-Dorfman disease with features of necrobiotic xanthogranuloma.

Case. A 45-year-old female presented with a 10-year history of an enlarging neck mass with normal overlying skin accompanied by dysphagia and multiple asymptomatic pink to yellowish-brown papules, nodules, and plaques on the face, trunk and extremities. Biopsies of a skin nodule and plaque revealed granulomatous dermal infiltrates (lymphocytes, foamy histiocytes, and Touton giant cells), emperipolesis and areas of necrosis. CD1A and Fite-Faraco staining showed negative results while CD68 and S100 positively stained the tissues of interest. Histopathology of the neck mass paralleled these findings in addition to being negative for lymphoid markers. Patient had monoclonal gammopathy and thyromegaly with enlarged cervical lymph nodes on further tests and imaging. Intralesional and systemic steroids were given which led to flattening of skin lesions and improvement in dysphagia, respectively.

Conclusion. Diagnosis and classification of a particular type of non-LCH may be difficult due to similarities across its subtypes. Hence, it is our belief that these diseases may occur on a spectrum. Treatment involves a multidisciplinary approach for the best possible care.
\end{abstract}

Key Words: histiocytosis, non-Langerhans cell histiocytosis, Rosai-Dorfman disease, necrobiotic xanthogranuloma, case report

\section{INTRODUCTION}

Non-Langerhans cell histiocytosis is a group of diseases marked by proliferation of histiocytes other than Langerhans cells. We report the case of a patient with clinical and histopathological findings compatible with both RosaiDorfman disease (RDD) and necrobiotic xanthogranuloma (NXG). In global literature, there are 100 cases of NXG, mostly occurring between ages 17-85 years. A US registry reported 423 cases of RDD with an increase of 100 cases per year. Classical RDD was found to be more common among African males while the cutaneous form was more common in Asian females between $1^{\text {st }}$ and $2^{\text {nd }}$ decades of life. ${ }^{1}$ Local data from the Philippine Dermatological Society Health Information System (PDS-HIS) showed three cases each of NXG and RDD from 2011 to 2018. Shared features across the non- $\mathrm{LCH}$ have been reported worldwide; however there have been no other published case with features of both RDD and NXG. 

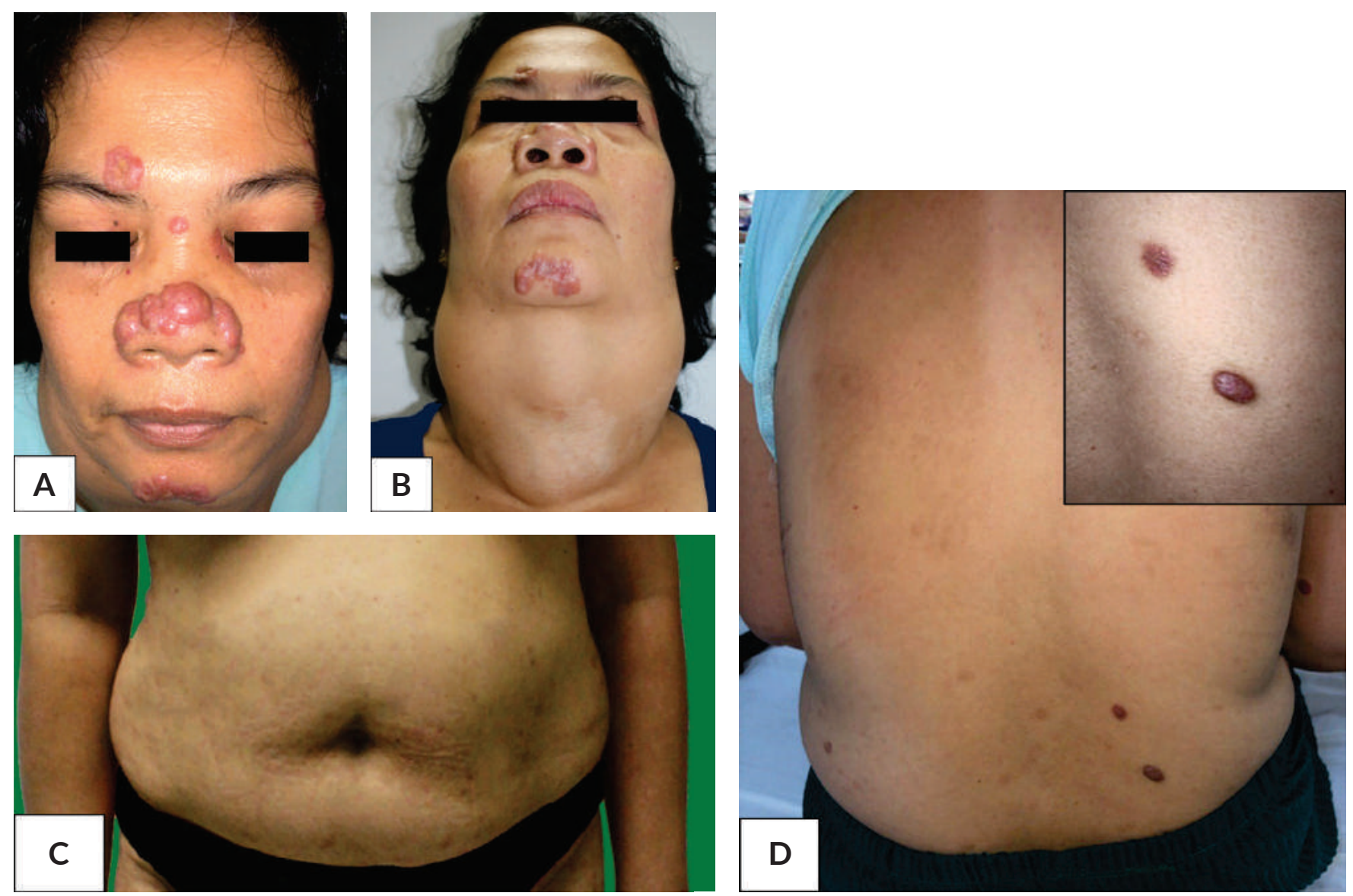

Figure 1. (A) Face: multiple, firm, erythematous to yellowish plaques and nodules, the largest (confluent nodules) measuring $0.3 \times 2 \times 6 \mathrm{~cm}$ (B) Large doughy neck masses. (C) Abdomen: multiple hyperpigmented macules. (D) Posterior trunk: multiple hyperpigmented and slightly erythematous macules with oval brown-red plaques; the largest measuring $1.1 \mathrm{x}$ $0.7 \times 0.4 \mathrm{~cm}$ (inset).

\section{CASE PRESENTATION}

A 45-year-old female came in with a 10-year history of an enlarging neck mass and asymptomatic pink-yellow papules and nodules on the face. Over the years, appearance of few brown to violaceous round and oval plaques on the trunk and extremities were noted, some eventually becoming arcuate with central clearing. On physical examination, there were multiple firm well-defined erythematous to yellowish plaques and nodules scattered around the eyebrows, medial canthi, nasal bridge and apex, and mentum (Figure 1A). Confluent, doughy non-tender masses were noted on the anterolateral aspects of the neck, measuring $18 \mathrm{~cm} \mathrm{x}$ $15 \mathrm{~cm} \times 12 \mathrm{~cm}$ (Figure 1B). Due to the mass, the trachea, cricoid, thyroid cartilage and the sternal notch were no longer palpable. No skin breaks or lesions were noted in the neck while the trunk showed several hyperpigmented and slightly erythematous macules with few brown-red oval plaques, especially at the lower back (Figure $1 \mathrm{C}$ and $\mathrm{D}$ ).

There were no palpable axillary or inguinal lymph nodes. The patient's upper extremities showed very few hyperpigmented macules and a solitary, non-tender, soft nodule on the right upper arm (Figure 2A); the lesion showed a yellowish core on dermoscopy (Figure 2A inset). On both lower extremities, there were multiple dusky and hyperpigmented macules and plaques; the larger lesions were of annular and arcuate morphology (Figure 2B).

The patient also complained of neck discomfort/ immobility, slight dysphagia and difficulty of breathing when lying supine. There were no fever, anorexia, weight changes, nor night sweats. Her medical history included cataract of both eyes, s/p phacoemulsification of the right, dyslipidemia, and cerebrovascular infarct. These left her with a leftsided blurred vision and left-sided weakness. The patient's family and social history were both non-contributory.

Blood work up were unremarkable except for slightly prolonged prothrombin time and dyslipidemia. ECG showed normal results. Neck and chest CT scan revealed an enlarged thyroid with several internal punctate calcifications noted within the right lobe. The trachea, esophagus, and vessels, such as the jugular veins and common carotid arteries, were displaced and compressed but not infiltrated. 

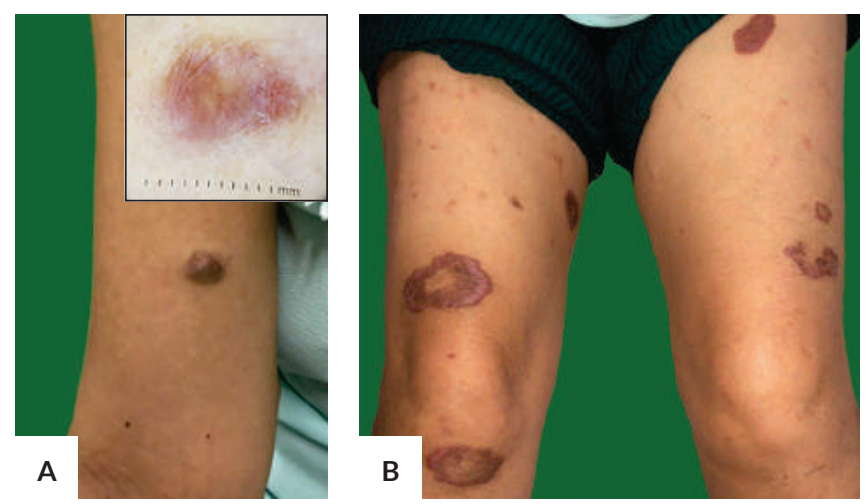

Figure 2. (A) Upper arm, right: solitary red-brown nodule measuring $1.2 \times 1 \mathrm{~cm}$. Inset photo: dermoscopic finding of few scales on an erythematous plaque with a yellowish core. (B) Lower extremities: multiple dusky and hyperpigmented macules and plaques, exhibiting annular and arcuate morphology.
Enlarged lymph nodes were also noted along the bilateral jugular chains.

Biopsies of two skin sites (nodule on the upper arm and arcuate plaque on the lower extremity) both revealed granulomatous dermal infiltrates containing lymphocytes, epithelioid cells, foam cells, and Touton giant cells. The sections showed emperipolesis as well as areas of necrosis intervening between the clusters of granuloma (Figure 3A to $3 \mathrm{C})$. No acid-fast bacilli were detected on Fite-Faraco stain and CD1A stain for Langerhans cells (Figure 4) revealed their normal presence in the epidermis. CD68 stain confirmed the presence of numerous histiocytes in the dermis (Figure 5) while S100 stain was positive and also demonstrated presence of emperipolesis (Figure 6A and B). Finally, Verhoeff-van Gieson stain revealed degenerated collagen and decreased amount of elastic fibers (Figure 7).

Incision biopsy of the anterior neck mass showed similar granulomatous infiltrates with emperipolesis consistent
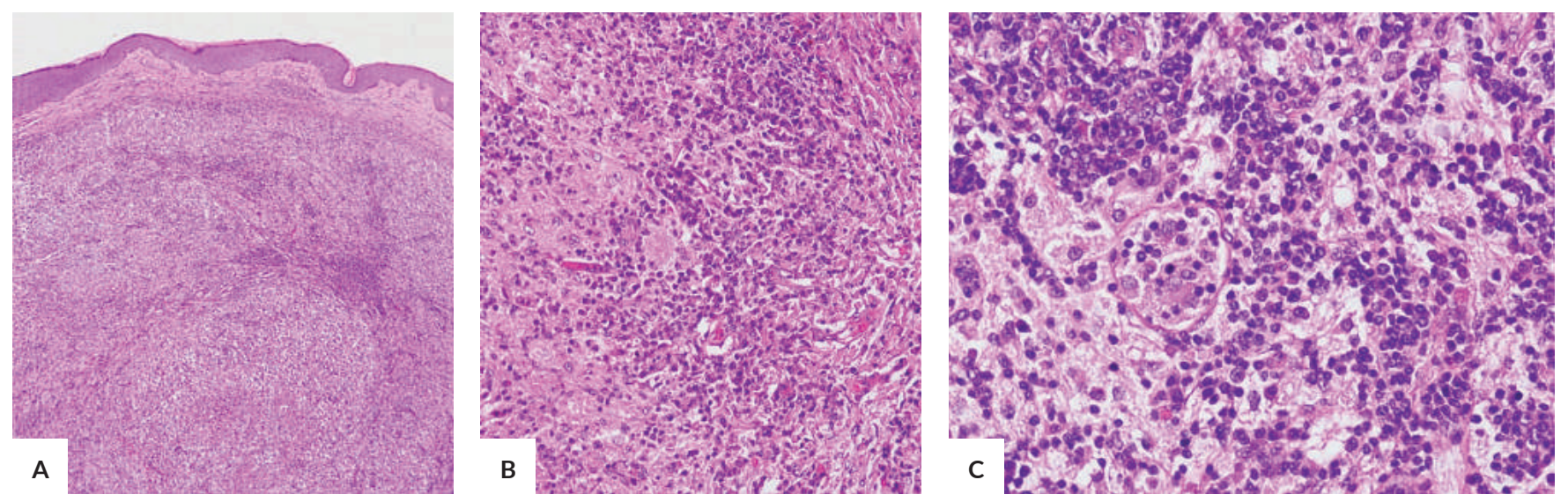

Figure 3. (A) Granulomatous infiltrates are seen involving the entire dermis with areas of degenerated collagen (necrosis) (Hematoxylin \& eosin, H\&E, X4). (B) Closer view of foamy histiocytes and Touton giant cell (H\&E, 40X). (C) Emperipolesis (H\&E, 40X).

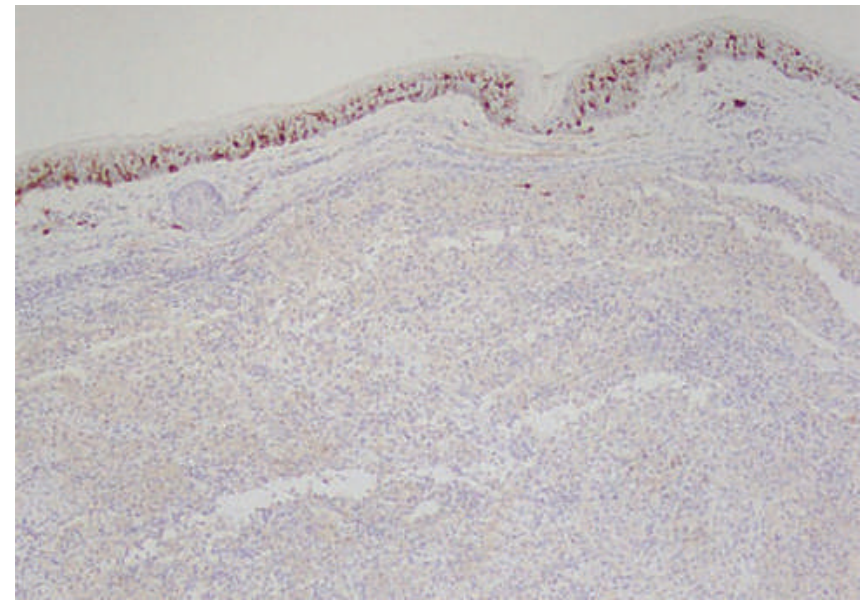

Figure 4. Langerhans cells limited to the epidermis (CD1A, 10X).

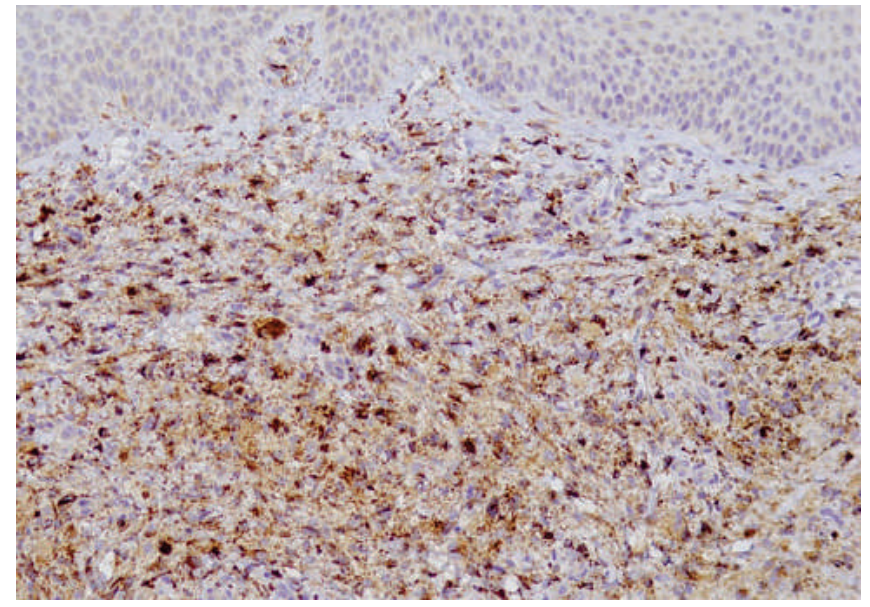

Figure 5. Numerous histiocytes confirmed with CD68 cytoplasmic staining in the dermis (CD68, 40X). 

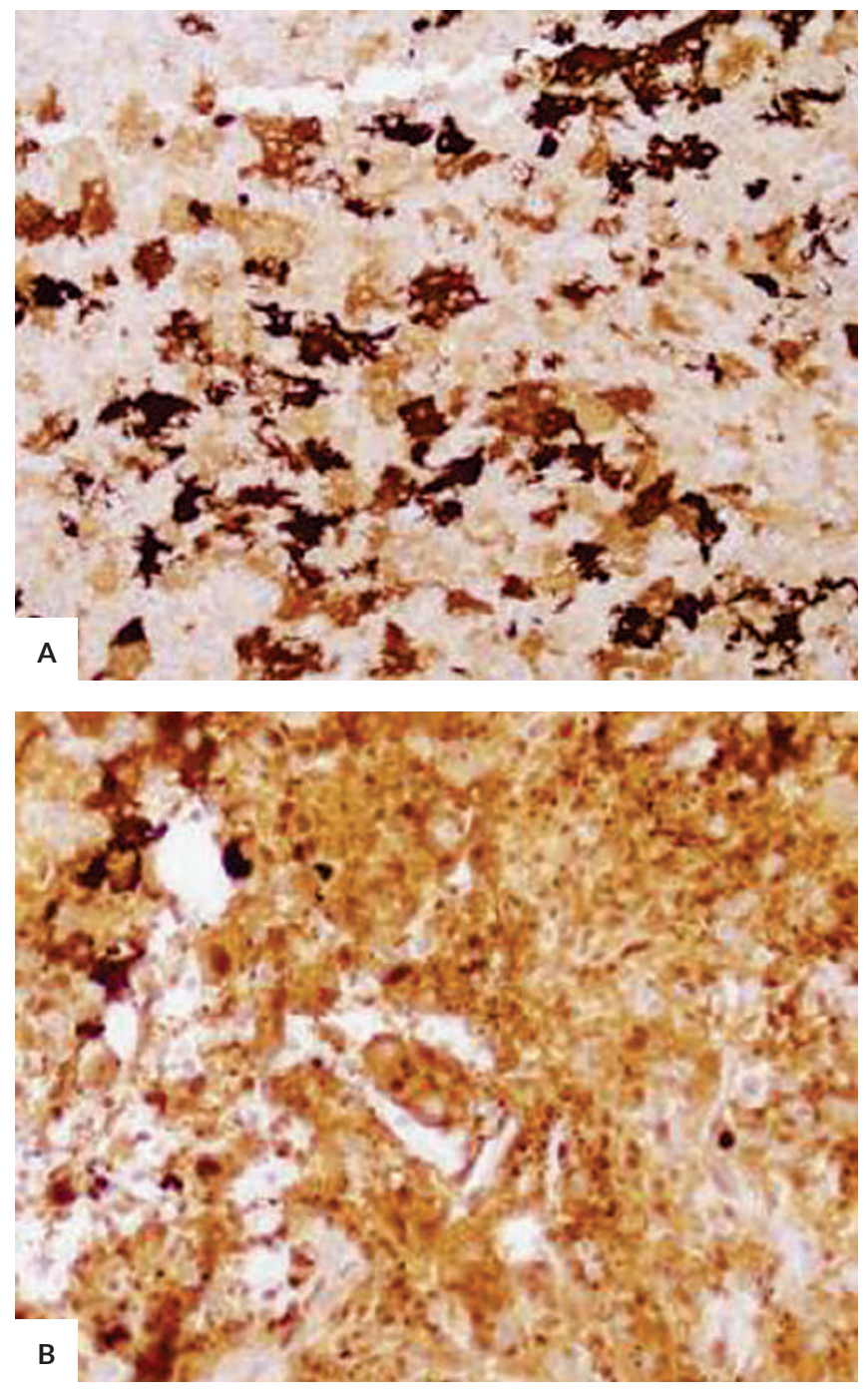

Figure 6. (A) Positive staining of giant cells (S100, 40X). (B) Emperipolesis (S100, 40X).

with Rosai-Dorfman disease. As with the skin biopsy, CD68 and S100 stained the tissues of interest; CD1A, CD3, CD20, CD30, Pancytokeratin and ALK were negative. No acid-fast bacilli were noted on auramine-rhodamine staining.

Assimilation of all the patient's signs, symptoms, and tests led us to the diagnosis of Rosai-Dorfman disease with features of necrobiotic xanthogranuloma. Based on this assessment, further hematologic tests were performed to evaluate associated conditions. Bone marrow aspiration and biopsy showed hypercellular marrow (70-80\% cellularity) with trilineage hematopoiesis without evidence of plasma cell neoplasm. There was no evidence of clonal aberrations on karyotyping. On serum protein electrophoresis, a monoclonal band was seen at the gamma region (37.5\%) relative to the other proteins, which was consistent with a monoclonal gammopathy. Systemic involvement was also demonstrated on positron emission tomography $(\mathrm{PET}) / \mathrm{CT}$ as hypermetabolic areas in the cervical and

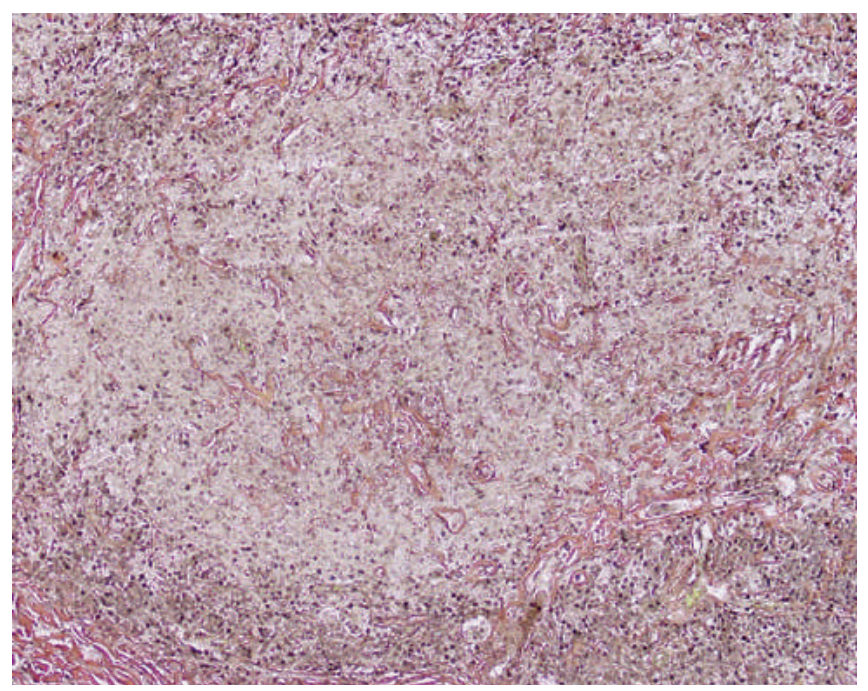

Figure 7. Walled-off area of the foamy histiocytes and necrosis showing degenerated collagen and decreased staining of elastic fibers (Verhoeff-van Gieson stain, 40X).
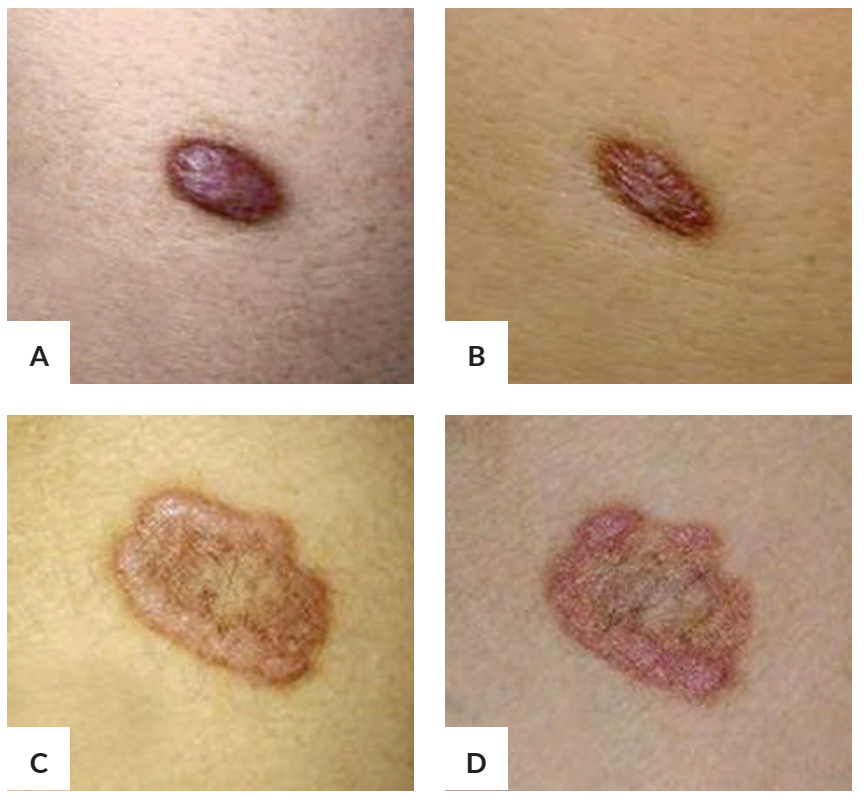

Figure 8. Notable flattening of the lesions at the posterior trunk $(A, B)$ before and 1 month after intralesional triamcinolone $20 \mathrm{mg} / \mathrm{ml}$; and at the thigh (C,D) before and 1 month after intralesional triamcinolone $10 \mathrm{mg} / \mathrm{ml}$.

tracheoesophageal lymph nodes, thyroid gland, multiple nasal polyps, cutaneous and subcutaneous lesions in the right frontal region, upper and lower extremities, and around the pelvis.

Intralesional triamcinolone $(40 \mathrm{mg} / \mathrm{ml})$ was attempted for the few larger discrete nodules and plaques on the back and thigh, with significant decrease in thickness after one session (Figure 8). 
Due to worsening of dysphagia and limitation of range of movement of the neck, patient was also given systemic steroids, dexamethasone $20 \mathrm{mg} /$ day for 5 days every 21-28 days, for a total of 6 cycles. There was no change in the size of cutaneous lesions and anterior neck mass; however, subjective improvement of dysphagia and range of motion of the neck were reported.

\section{DISCUSSION}

\section{Mononuclear Phagocytic System}

The mononuclear phagocytic system (MPS) is composed of monocytes, macrophages, and dendritic cells (DC). The system's nomenclature, however, is under constant examination as the system has been proposed to be dynamic with different and redundant origins of cells. ${ }^{2}$ As part of the MPS, histiocytes and disorders thereof, are better understood after examination of their origin and function.

Macrophages and dendritic cells are the major cell lines involved in histiocytosis. ${ }^{3}$ The process begins as hematopoietic stem cells in the bone marrow progressively commit and develop into monocytes, one of the early responders to infection. As these monocytes migrate out of the bone marrow and travel to the bloodstream, their expression of surface markers CD14 and CD16 determine their classification into classical, non-classical, and intermediate types. ${ }^{4}$ Based on different stimuli, classical monocytes travel into tissues and become macrophages, acquiring different names along the way, such as osteoclasts in bone, microglial cells in the central nervous system, and histiocytes in the connective tissues, among others. Thus, they are able to replenish macrophages and DC populations in different tissues. ${ }^{4,5}$

Macrophages/histiocytes may normally increase as a form of physiologic response to inflammation. However, pathologic excess as a response to infection, foreign body, and inflammation/necrosis may lead to histiocytosis. ${ }^{2}$

\section{Classification}

A diverse group of diseases, histiocytosis, have been classified many times in the past, with attempts being said to be unsatisfactory. ${ }^{6}$ The first classification of histiocytoses was by the Working Group of the Histiocyte Society in 1987; this only included Langerhans cell-related, non-Langerhans cell-related, and malignant histiocytosis. With improved understanding of these diseases, Emile and colleagues have proposed a revision of classification in 2016 based on clinical findings, histology, imaging and molecular features. ${ }^{7}$

\section{Diagnosis}

Non-LCH is mainly diagnosed according to immunohistochemistry findings. On histology, well-circumscribed nodules containing histiocytes are located in the dermis with inflammatory cells surrounding vessels and the lesion. ${ }^{8}$ Diagnosis may be difficult as characteristics are overlapping within the non-LCH groups. Nevertheless, with the salient

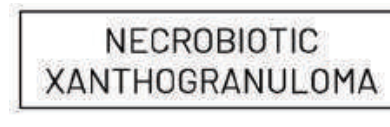

ROSAI-DORFMAN DISEASE

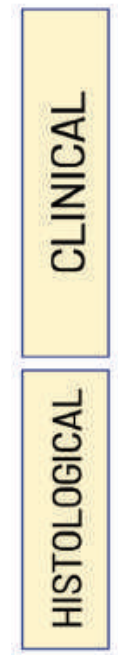

Asian female

Cervical lymphadenopathy

\section{Periorbital lesions}

Chronicity

Paraproteinemia

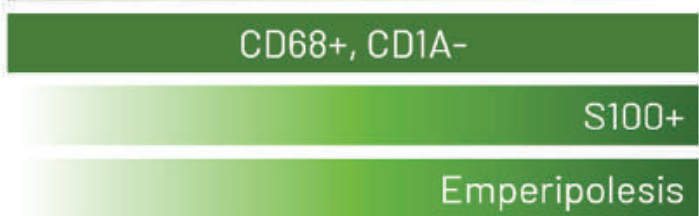

Necrosis

Figure 9. Patient's features are shown in gradient or solid fill to represent which disease it is more commonly associated with. Lighter fill: less commonly seen in the disease; darker fill: more commonly seen in the disease; solid fill: may equally be found in both/no predilection.

features presented in this case, the patient's condition was narrowed down to two non-LCH entities-NXG and RDD. A diagram of the patient's features, signs, and symptoms are shown in Figure 9. These features, however, are not solely found or exclusive to one disease.

RDD is a mostly self-limited disease which belongs to the $\mathrm{R}$ group (classical) and the $\mathrm{C}$ group (cutaneous) of the 2016 classification. The classical (nodal) form, more commonly seen in male children and young adults, presents with cervical lymphadenopathy and extra-nodal disease affecting skin, bone, and meninges. Meanwhile, the cutaneous form, mostly in Asian females, present with a wide range of skin lesions such as yellow macules and patches to red-brown papules, plaques and nodules, which may develop erosion/ulceration. ${ }^{9}$ A literature review by Al-Khateeb showed that these lesions are found on the face in $11.2 \%$ of cases; while in the body, several were found in distal extremities. ${ }^{10}$

The etiology of RDD is poorly understood. Some authors have associated it with viral infections (herpes, cytomegalovirus, and HIV) and with missense mutations in kinases (NRAS, $K R A S$, and $A R A F))^{1,11}$ suggesting a reactive and/or clonal process. ${ }^{11,12}$ Histologically, both nodal and extra-nodal sites may have areas of pale cells with surrounding lymphocyte- and plasma cell-rich zones. The hallmark of RDD is emperipolesis, defined as inflammatory cells (lymphocytes or neutrophils) being engulfed by histiocytes; the degree of which can be variable., ${ }^{2,13}$ Associated diseases include autoimmune diseases (e.g., systemic 
lupus erythematosus, autoimmune hemolytic anemia), malignancy (e.g., lymphomas, myelodysplastic syndrome, malignant histiocytosis), and other inherited disorders. ${ }^{1}$ Our patient profile of being an Asian female, having cervical lymphadenopathy, cutaneous lesions, and emperipolesis on histology are compatible with both classical and cutaneous RDD.

On the other hand, NXG belongs to the C group and is a chronic granulomatous disease which commonly involves the periorbital area and later proceeds to affect the trunk and limbs. ${ }^{14,15}$ Its exact etiopathogenesis is unknown but the presence of faulty phagocytic mechanisms and granulomatous response to paraprotein-lipid complex in the skin have been speculated. ${ }^{16,17}$ The appearance of multiple asymptomatic yellow-to-reddish brown papules and nodules that later develop into plaques are characteristic of NXG. Ulceration, telangiectasia and atrophy of lesions are also expected. ${ }^{9}$

Histologically, NXG has a normal or atrophic epidermis and granulomas consisting of foamy histiocytes, lymphocytes, and giant cells; a palisade is formed around areas of necrosis which sometimes contain cholesterol crystals. ${ }^{15,18}$ NXG has been associated with paraproteinemia, monoclonal gammopathy, multiple myeloma, and amyloidosis., ${ }^{6,19}$ In fact, $80 \%$ of NXG patients have monoclonal gammopathy, usually with immunoglobulin $G \kappa$ and $\lambda$ type. ${ }^{13}$ In our case, the transition from papules and nodules to plaques was noted, although the lesions were not concentrated primarily on the periorbital area. The chronicity of her condition ( 10 years), necrosis on histology, and association with monoclonal gammopathy strongly suggest coexistence of NXG.

Being part of the non-LCH disorders, both RDD and NXG are positive for CD68 stain and negative for CD1A stain. Emperipolesis and positivity for S100 stain is seen in RDD while necrosis is more often found in NXG. ${ }^{9}$ Coexistence of two non- $\mathrm{LCH}$ disorders as well as with an $\mathrm{LCH}$ disorder in the same patient have also been described in the past. ${ }^{20}$ In one study with combined RDD and LCH, RDD histiocytes were postulated to have undergone clonal transformation and phenotypic switching to $\mathrm{LCH}$ as suggested by presence of clonal abnormalities and genetic gains/losses across the disorders. ${ }^{21}$ The concurrent presence of JXG and RDD has also been reported in a Thai boy; however, the relationship of the two diseases has remained unexplored..$^{22}$ The overlapping clinical and histopathological features discussed reinforce the existence of a spectrum across the histiocytic disorders and emphasize the importance of balancing strong clinical judgment with histopathologic assessment as well as keeping flexible differential diagnosis.

A notable differential diagnosis in this case is necrobiosis lipoidica, which, like NXG, may initially present as papules and nodules that eventually flatten, creating an atrophic center. It has histologic similarities with NXG which can cause quite the challenge in diagnosis. ${ }^{15}$ Infiltrates are composed mostly of lymphocytes and plasma cells with some histiocytes and giant cells; necrosis is seen in layers that alternate with the said infiltrates. ${ }^{19,23}$

\section{Management and Prognosis}

NXG is a chronic disease where new lesions may develop throughout the course; response to treatment is variable. ${ }^{15}$ Extensive involvement of the orbital region may lead to ocular complications such as blurry vision, diplopia, keratitis and corneal perforation. ${ }^{24} \mathrm{RDD}$ is self-limited; however, those with multiorgan involvement and immune dysfunction have poorer prognosis and may progress and lead to death..$^{25}$

Treatment algorithms for both NXG and RDD are available. ${ }^{1,15}$ These include surgical resection for localized lesions, intralesional/systemic corticosteroids, and chemotherapy using melphalan, chlorambucil, and cyclophosphamide, among others. However, it should be noted that due to the rarity of non- $\mathrm{LCH}$, there is no one standard thera-peutic option. In our case, a trial of intralesional triamcinolone and oral dexamethasone afforded some improvement and relief from symptoms. Other cases of NXG and RDD in the literature showed variable, partial, and good responses after treatment with intralesional and/ or oral corticosteroids (dexamethasone and prednisone/ prednisolone). ${ }^{15}$ Relapses, however, may be possible with treatment interruptions, ${ }^{1}$ hence regular follow-up consultations are advised.

\section{CONCLUSION}

The overlapping features seen across non-LCH subtypes reflect the existence of a spectrum, making a straightforward diagnosis challenging. We presented a case of an adult female with features of both RDD and NXG that showed some improvement with intralesional and systemic corticosteroids. Regular follow-up consultations with a multidisciplinary team are ideal to monitor for complications and progression of disease.

\section{Acknowledgment}

The authors thank Dr. Jolene Gatmaitan-Dumlao (Dermatopathology Fellow) and Dr. Carlo Miguel Berba (Oncology Fellow).

\section{Statement of Authorship}

All authors have substantial contributions to acquisition, analysis, interpretation of data; drafting the work; approval of the final version; and agreement to be accountable for all aspects of the work.

\section{Author Disclosure}

All authors declared no conflicts of interest.

\section{Funding Source}

No funding support. 


\section{REFERENCES}

1. Abla O, Jacobsen E, Picarsic J, Krenova Z, Jaffe R, Emile J-F, et al. Consensus recommendations for the diagnosis and clinical management of Rosai-Dorfman-Destombes disease. Blood. 2018 Jun 28; 131(26):2877-90.

2. Picarsic JL, Chikwava K. 19 - Disorders of Histiocytes**The authors gratefully acknowledge the contribution of Dr. Ronald Jaffe, who authored this chapter in previous editions and critically reviewed the current edition. In: Hsi ED, editor. Hematopathology (Third Edition) [Internet]. Philadelphia: Content Repository Only!; 2018 [cited 2020 Jun 18]. p. 567-616.e4. (Foundations in Diagnostic Pathology). Available from: http://www.sciencedirect.com/science/ article/pii/B9780323479134000197

3. Ratzinger G, Zelger BWH. Juvenile xanthogranuloma and other non-Langerhans cell histiocytoses. Harper's Textbook of Pediatric Dermatology. 2019 Nov 20;1078-96.

4. Castagna A, Polati R, Bossi AM, Girelli D. Monocyte/macrophage proteomics: recent findings and biomedical applications. Expert Rev Proteomics. 2012 Apr;9(2):201-15.

5. Narasimhan PB, Marcovecchio P, Hamers AAJ, Hedrick CC. Nonclassical monocytes in health and disease. Annu Rev Immunol. 2019 Apr 26; 37:439-56

6. Classen CF, Minkov M, Lehrnbecher T. The non-Langerhans cell histiocytoses (Rare histiocytoses) - Clinical Aspects and Therapeutic Approaches. Klin Padiatr. 2016 Nov; 228(6-07):294-306.

7. Emile J-F, Abla O, Fraitag S, Horne A, Haroche J, Donadieu J, et al. Revised classification of histiocytoses and neoplasms of the macrophage-dendritic cell lineages. Blood. 2016 Jun 2; 127(22): 2672-81.

8. Weitzman SS, Jaffe RM. Uncommon histiocytic disorders: the non-Langerhans cell histiocytoses. Pediatric blood \& cancer. 2005; 45(3):256-64.

9. Gelmetti C. Chapter 148. Non-Langerhans Cell Histiocytosis. In: Fitzpatrick's Dermatology in General Medicine [Internet]. 8th ed. New York: McGraw-Hill Medical; 2012 [cited 2019 Sep 9]. p. 1795808. Available from: https://accessmedicine.mhmedical.com/content. aspx? bookid $=392 \&$ sectionid $=41138870 \&$ jumpsectionid $=41158230$

10. Al-Khateeb T. Cutaneous Rosai-Dorfman Disease of the Face: A Comprehensive Literature Review and Case Report. J Oral Maxillofac Surg. 2016 Mar; 74(3):528-40.

11. Garces S, Medeiros LJ, Patel KP, Li S, Pina-Oviedo S, Li J, et al. Mutually exclusive recurrent KRAS and MAP2K1 mutations in Rosai-Dorfman disease. Mod Pathol. 2017; 30(10):1367-77.

12. Shanmugam V, Margolskee E, Kluk M, Giorgadze T, Orazi A. RosaiDorfman disease harboring an activating KRAS K117N missense mutation. Head Neck Pathol. 2016 Sep; 10(3):394-9.
13. Schmieder A, Goerdt S, Utikal J. Chapter 117. Histiocytosis. In: Fitzpatrick's Dermatology. 9th ed. New York: McGraw-Hill Education; 2019. p. 2018-41.

14. Girisha BS, Holla AP, Fernandes M, Noronha TM. Necrobiotic Xanthogranuloma. J Cutan Aesthet Surg. 2012; 5(1):43-5.

15. Miguel D, Lukacs J, Elsner P, Illing T. Treatment of necrobiotic xanthogranuloma - a systematic review. JEADV. 2017; 31:221-35.

16. Schuering R,Morgan MB. Lethal Non-Langerhans Cell Histiocytoses: Necrobiotic Xanthogranuloma and Xanthoma Disseminatum. In: Crowe DR, Morgan M, Somach S, Trapp K, editors. Deadly Dermatologic Diseases: Clinicopathologic Atlas and Text [Internet]. Cham: Springer International Publishing; 2016 [cited 2020 Jun 26]. p. 129-33. Available from: https://doi.org/10.1007/978-3-31931566-9_20

17. Flann S, Wain EM, Halpern S, Andrews V, Whittaker S. Necrobiotic xanthogranuloma with paraproteinaemia [Internet]. Clinical and Experimental Dermatology. 2006 [cited 2019 Aug 21]. Available from: https://onlinelibrary.wiley.com/doi/abs/10.1111/ j.1365-2230.2005.02042.x

18. Elner VM, Mintz R, Demirci H, Hassan AS. Local corticosteroid treatment of eyelid and orbital xanthogranuloma. Trans Am Ophthalmol Soc. 2005 Dec; 103:69-74.

19. Luz FB, Gaspar AP, Kalil-Gaspar N, Ramos-e-Silva M. Histiocytes and non-Langerhans cell histiocytoses in dermatology. An Bras Dermatol. 2003 Feb; 78(1):99-118.

20. Ruby K, Deng A, Zhang J, LeBlanc R, Linos K, Yan S. Emperipolesis and S100 positivity may be seen in cutaneous xanthogranulomas: a multi-institutional observation. J Cutan Pathol. 2018 May 24; 45.

21. O'Malley DP, Duong A, Barry TS, Chen S, Hibbard MK, Ferry JA, et al. Co-occurrence of Langerhans cell histiocytosis and RosaiDorfman disease: possible relationship of two histiocytic disorders in rare cases. Mod Pathol. 2010 Dec;23(12):1616-23.

22. Poklang V, Shuangshoti S, Chaipipat M, Nuchprayoon I, Shuangshoti S. Rosai-Dorfman disease and juvenile xanthogranuloma in a Thai boy: report of a case. J Med Assoc Thai. 2006 Jan; 89(1):94-8.

23. Tallon B. Necrobiosis lipoidica pathology [Internet]. DermNet. 2011 [cited 2019 Sep 14]. Available from: https://www.dermnetnz. org/topics/necrobiosis-lipoidica-pathology/

24. Balagula Y, Straus DJ, Pulitzer MP, Lacouture ME. Necrobiotic Xanthogranuloma Associated With Immunoglobulin M Paraproteinemia in a Patient With Waldenström Macroglobulinemia. JCO. 2011 Jan 18; 29(11):e305-7.

25. Miękus A, Stefanowicz J, Kobierska-Gulida G, AdamkiewiczDrożyńska E. Rosai-Dorfman disease as a rare cause of cervical lymphadenopathy - case report and literature review. Cent Eur J Immunol. 2018; 43(3):341-5. 